\title{
Modeling of Medium-Voltage Power-Line Communication Systems Noise Levels
}

\author{
Apostolos N. Milioudis, Student Member, IEEE, Konstantinos N. Syranidis, Georgios T. Andreou, Member, IEEE, \\ and Dimitrios P. Labridis, Senior Member, IEEE
}

\begin{abstract}
The implementation of power-line communication systems requires detailed knowledge of the channel properties, such as transfer function, noise levels, and channel capacity in order to assess the services that can be provided. In this paper, the interference scenario on overhead medium-voltage power distribution lines caused by external electromagnetic (EM) fields is examined by focusing on the noise induced. The external EM fields are considered to be the main source of occurring noise and a novel method capable to calculate the corresponding noise levels is presented. The proposed method is compared to other existing models and its strengths are highlighted. The effect of the induced noise to the overall data capacity of the power-line communication channel is examined. Eventually, the influence of several parameters, such as the magnitude and direction of propagation of the external EM field, network topology, earth's EM properties, and different levels of emissions suggested by various organizations are investigated.
\end{abstract}

Index Terms-Broadband over power lines (BPL), channel capacity, coupled mode analysis, electromagnetic coupling, electromagnetic fields, electromagnetic interference, modal analysis, noise calculation, overhead power lines, power distribution lines, powerline communications (PLC).

\section{INTRODUCTION}

$\mathbf{T}$ HE ever-increasing necessity for data transfer, due to the constant development of communication systems, leads to the boost of economical feasible technologies, such as powerline communications (PLC). The exploitation of the particular technology appears to be tempting, because it uses the existing electrical power grid infrastructure, and does not require further investment for backbone installation. The upcoming upgrade of the traditional power grids to smart grids, which will incorporate information and communication technology (ICT) toward improving their functionality, offers a unique opportunity for the PLC technology to be implemented in large scale for the transmission of network operation-related data [1]. Several models proposing fault isolation, self healing, and online measurements at various points of the network can be incorporated in the new, smart era of the grid, and PLC systems could be utilized for the respective data transfer.

However, power grids were designed to deliver electric power to consumers at very low frequencies $(50$ or $60 \mathrm{~Hz})$ and

Manuscript received February 14, 2012; revised January 22, 2013 and March 05, 2013; accepted April 12, 2013. Date of publication June 19, 2013; date of current version September 19, 2013. This work was supported in part by the Greek State Scholarships Foundation (SSF). Paper no. TPWRD-00149-2012.

The authors are with the Department of Electrical and Computer Engineering, Aristotle University of Thessaloniki, Thessaloniki 54124, Greece (e-mail: amilioud@auth.gr; ksyranid@gmail.com; gandreou@auth.gr; labridis@auth.gr).

Digital Object Identifier 10.1109/TPWRD.2013.2258408 not to carry frequencies in the range of megahertz, implemented by PLC technology. Furthermore, the networks typically spread to vast areas and contain many lateral branches. Thus, PLC systems suffer from attenuation and multipath propagation, problems similar to those appearing in wireless communication systems [2]-[5]. Because of this, such a system requires a rigorous study prior to its installation, in order to determine all required parameters [6]-[11]. A key feature that may determine the penetration level of this technology is the data capacity of the PLC communication channels. This mainly depends on the available frequency range, transmitted power, channel's transfer function, and existing noise. Each of these factors can drastically enhance or deteriorate the channel performance. Studies have been conducted aiming mainly on the effect of the first three factors on the capacity of PLC channels [12]-[15], but the influence of the channel noise also has to be taken into account.

PLC channel noise is influenced mainly by the voltage level, the characteristics of the power grid appliances, the features of the transmission line, and the electromagnetic (EM) activity in the vicinity of the communication channel. In addition, it can be divided into two main categories, defined here as endogenous and exogenous noise, representing the self-imposed and the induced noise, respectively. The former is caused by the function of the power grid, whereas the latter exists mainly due to external EM fields in the vicinity of the power lines inducing noise due to the fact that power cable installations are unshielded.

Models existing in the literature deal with the issue of noise modeling by incorporating random peaks of noise that can be caused by interference [2], [15], or assuming a constant noise level over the used frequency range [12], [14]. Both aforementioned models do not take into account several important characteristics of the network and cannot be applied in every case. Furthermore, measurements have been conducted for determining the existing noise level in MV networks [16], [17], and studies have been conducted for the impulsive noise modeling [18]-[20].

In this study, a novel approach considering external EM activity in the vicinity of the arrangement as the main source of noise on overhead MV transmission lines, which induces noise on the network, is presented. Therefore, a thorough investigation concerning the effect of this external EM field on the induced noise and the channel data capacity are presented. In addition, the influence of various features, such as EM-field magnitude and orientation, different emission levels as proposed by various countries and organizations, the network topology, and earth's EM properties are examined. The proposed method could be very helpful during the study preceding the installa- 
tion of a broadband PLC system at an overhead MV line. The external EM activity of the region in which the transmission line is installed can be used in order to calculate the corresponding noise levels. These can be used for the computation of the channel's expected capacity and, thus, the services that can be successfully provided by the PLC system can be determined.

In Section II, the origin of noise in aerial MV networks is examined and its significant features are explained. Subsequently, Section III contains the theoretical analysis required for the calculation of the induced noise on the network. All of the conducted simulations and the respective results and conclusions are shown in Sections IV and V, respectively.

\section{ORIGIN OF NOISE IN AERIAL MV Networks}

The noise occurring in power networks may cause problems and malfunction to an installed communication system, such as high bit-error rate (BER) or burst errors. For this reason, a comprehensive investigation of the noise origin is indispensable. Furthermore, it is important to focus on the specific attributes of the studied part of the network (i.e., the MV network).

In general, possible noise origins may be divided into two main categories: corresponding to sources or events that are either endogenous or exogenous. Endogenous is in reference to the sources that are part of the MV network, such as electrical equipment, whereas exogenous refers to the sources that do not constitute part of the power network and do not participate in its operation.

In more detail, endogenous noise could be generated by electrical devices, switching operations, network faults, and spark discharges since they are defined in [21]. The MV part of the network is separated from the low voltage (LV) and the high voltage (HV) parts by power transformers. These are considered to behave as open circuits at the frequency range utilized by the PLC technology, providing complete isolation from the rest of the network. Devices that are connected directly to the MV network could be capacitor banks and motors that do not produce noise at the studied range. The rest of the equipment is connected through a power transformer, leading to negligible noise production. Furthermore, it can be considered that both switching operations and faults would lead to burst errors, making communication impossible during their occurrence, but their appearance is rare. Moreover, spark discharges do not occur on all power lines and that it is why these types of discharges are not frequently documented in the literature. In fact, their appearance could be considered rare and does not correspond to normal operating conditions. They are caused by bad connections between conductors and insulators, caused by oversight during the installation process, that does not constitute the usual case. The existence of spark discharges could create problems to PLC system operation due to their spectral content. For that reason, it is important that bad connections creating them are fixed prior to PLC system installation. Considering that a functioning PLC system and spark discharges will not coexist, this particular source of noise is neglected. Conclusively, all possible endogenous noise origins may be omitted in a noise calculating model.

Possible origins of exogenous noise are external EM fields and weather conditions. The former may be transmitted by another communication system utilizing the same frequency range and inducing thus noise on the studied PLC system due to the absence of cable shielding. The latter may cause the initiation of corona discharges that are reported as possible noise sources and atmospheric noise. Specifically, regarding corona discharges, the threshold value of the electric-field strength for corona phenomenon initiation, as computed using Peek's equation [22] under normal weather conditions (i.e., air pressure of $760 \mathrm{mmHg}$, temperature of $20{ }^{\circ} \mathrm{C}$ ) for a typical overhead MV line with stranded aluminum conductor steel-reinforced (ACSR) conductors of $70 / 12 \mathrm{~mm}^{2}$ is close to $36 \mathrm{kV} / \mathrm{cm}$, while the maximum calculated electric-field strength for a typical overhead 20-kV MV line is approximately $6 \mathrm{kV} / \mathrm{cm}$. By comparing these values, it can be deducted that corona discharges would only manifest themselves in MV power lines under extremely severe weather conditions. Furthermore, atmospheric noise that could be created by lightning could raise the noise levels, in some cases so high that communication could become infeasible. However, the existence of lightning-induced atmospheric noise can be considered as rarely appearing and is chosen to be omitted. Hence, the main origin of noise generation for the system investigated in the current work is the external EM fields.

\section{THEORETICAL FORMULATION}

\section{A. Interference on the Transmission Line Caused by an External EM Field}

The effect of an external EM field on a transmission line may be expressed using field-to-transmission-line coupling equations formed similar to the telegraph equations for voltage and current propagation, such as those proposed by Agrawal et al. [23]-[25], shown in (1) and (2)

$$
\begin{aligned}
& \frac{\partial \mathbf{V}^{\mathbf{s}}(\mathbf{x})}{\partial x}+\mathbf{Z}^{\prime} \mathbf{I}(\mathbf{x})=\mathbf{E}_{\mathbf{x}}^{\mathbf{e}}(\mathbf{x}, \mathbf{h}) \mathbf{d} \mathbf{x} \\
& \frac{\partial \mathbf{I}(\mathbf{x})}{\partial x}+\mathbf{Y}^{\prime} \mathbf{V}^{\mathbf{s}}(\mathbf{x})=0 .
\end{aligned}
$$

The elements of the voltage vector $\mathrm{V}^{\mathbf{s}}(\mathrm{x})$ correspond to the line integrals of the scattered electric field between the ground and each line conductor, respectively. Moreover, $\mathbf{Z}^{\prime}$ and $\mathbf{Y}^{\prime}$ correspond to the per-unit length impedance and admittance matrices of the transmission line, respectively, and can be calculated as explained in [26]. Furthermore, vector $\mathbf{E}_{\mathbf{x}}^{\mathbf{e}}(\mathbf{x}, \mathbf{h}) \mathbf{d x}$ represents the external electric-field component parallel to the conductor's axis.

The analysis of the problem can be simplified by implementing the modal transformation technique, through which only $N$-independent modes may be considered. The voltage and current vectors of the arrangement are linked to their modal components through specific transformation matrices $\mathbf{T}_{\mathbf{V}}$ and $\mathbf{T}_{\mathbf{I}}$, as shown in (3) and (4), respectively [27]

$$
\begin{aligned}
\mathbf{V}^{\mathbf{s}}(\mathbf{x}) & =\mathbf{T}_{\mathbf{V}} \cdot \mathbf{V}_{\mathbf{m}}^{\mathbf{s}}(\mathbf{x}) \\
\mathbf{I}(\mathbf{x}) & =\mathbf{T}_{\mathbf{I}} \cdot \mathbf{I}_{\mathbf{m}}(\mathbf{x})
\end{aligned}
$$

With the application of the modal transformation, the field to transmission-line equations may be uncoupled as shown in (5) 


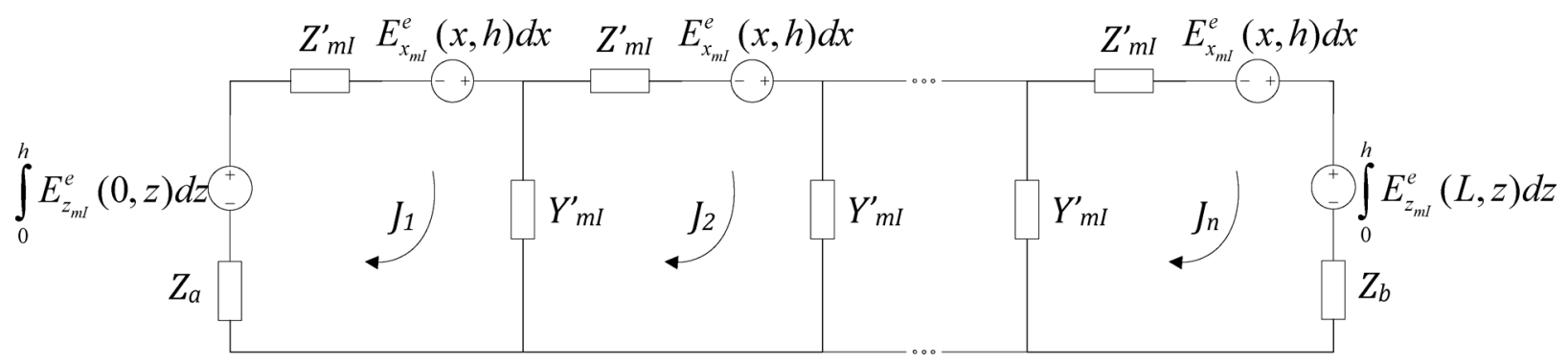

Fig. 1. Mesh current method for the $I^{\text {th }}$ mode of a topology with no lateral branches.

and (6). The transformation matrices $\mathbf{T}_{\mathbf{V}}$ and $\mathbf{T}_{\mathbf{I}}$ may be used to diagonalize the per-unit impedance and admittance matrices $\mathbf{Z}^{\prime}, \mathbf{Y}^{\prime}$, as shown in (7) and (8), leading to the analysis of completely independent circuit equivalents. Each of these equivalents corresponds to a specific mode, implementing the modal per-unit length impedance $Z_{m I}^{\prime}$ and admittance $Y_{m I}^{\prime}$ of the $I^{\text {th }}$ mode [28]. Moreover, the term $\mathbf{T}_{\mathbf{V}}^{-1} \cdot \mathbf{E}_{\mathbf{x}}^{\mathbf{e}}(\mathbf{x}, \mathbf{h}) \mathbf{d x}$ corresponds to the transformed vector $\mathbf{E}_{\mathbf{x}}^{\mathrm{e}}(\mathbf{x}, \mathbf{h}) \mathbf{d} \mathbf{x}$

$$
\begin{aligned}
& \frac{\partial \mathbf{V}_{\mathbf{m}}^{\mathbf{s}}(\mathbf{x})}{\partial x}+\mathbf{T}_{\mathbf{V}}^{-\mathbf{1}} \cdot \mathbf{Z}^{\prime} \cdot \mathbf{T}_{\mathbf{I}} \cdot \mathbf{I}_{\mathbf{m}}(\mathbf{x})=\mathbf{T}_{\mathbf{V}}^{-\mathbf{1}} \cdot \mathbf{E}_{\mathbf{x}}^{\mathbf{e}}(\mathbf{x}, \mathbf{h}) \mathbf{d} \mathbf{x} \\
& \frac{\partial \mathbf{I}_{\mathbf{m}}(\mathbf{x})}{\partial x}+\mathbf{T}_{\mathbf{I}}^{-\mathbf{1}} \cdot \mathbf{Y}^{\prime} \cdot \mathbf{T}_{\mathbf{V}} \cdot \mathbf{V}_{\mathbf{m}}^{\mathbf{s}}(\mathbf{x})=\mathbf{0} \\
& \mathbf{T}_{\mathbf{V}}^{-1} \cdot \mathbf{Z}^{\prime} \cdot \mathbf{T}_{\mathbf{I}}=\operatorname{diag}\left\{Z_{m 1}^{\prime} Z_{m 2}^{\prime} \cdots Z_{m N}^{\prime}\right\} \\
& \mathbf{T}_{\mathbf{I}}^{-\mathbf{1}} \cdot \mathbf{Y}^{\prime} \cdot \mathbf{T}_{\mathbf{V}}=\operatorname{diag}\left\{Y_{m 1}^{\prime} Y_{m 2}^{\prime} \cdots Y_{m N}^{\prime}\right\}
\end{aligned}
$$

\section{B. Noise Calculation at Topologies With No Lateral Branches}

Considering a transmission line of $N$ conductors and length $L$, located above a lossy ground plane, $N$ equivalent circuits, such as the one illustrated in Fig. 1, may be formed, each one corresponding to a specific mode. Each circuit incorporates the termination impedances $Z_{a}$ and $Z_{b}$, corresponding to the communication devices installed at the starting and ending point of the topology, respectively; the integral of the transformed $z$ component of the external electric field at both ends, and the transformed longitudinal voltage sources corresponding to the $x$ component of the external electric field. Applying the mesh current method to the circuit formed by $n$ loops [29], as shown in Fig. 1, gives (9). A corresponds to a $n \times n$ matrix and is derived as

$$
\begin{aligned}
& \mathbf{A} \cdot \mathbf{J}=\mathbf{E} \\
& \mathbf{A}=\mathbf{A}_{\mathbf{d}}+\mathbf{A}_{\mathbf{t}}+\left(\mathbf{A}_{\mathbf{t}}\right)^{t} .
\end{aligned}
$$

The elements of matrices $\mathbf{A}_{\mathbf{d}}$ and $\mathbf{A}_{\mathbf{t}}$ are defined in (11) and (12), respectively, where $T$ is a set containing the number of loops having a termination which, in the examined cases with no lateral branches, is equal to $\{1, n\}$, and $Z_{\text {term } i}$ is the corresponding terminating impedance of loop $i$

$$
A_{d i j}= \begin{cases}Z_{m I}^{\prime}+\frac{2}{Y_{m I}^{\prime}}, & \text { if } \mathrm{i}=\mathrm{j} \text { and } \mathrm{i} \notin \mathrm{T} \\ Z_{\mathrm{term} i}+Z_{m I}^{\prime}+\frac{1}{Y_{m I}^{\prime}}, & \text { if } \mathrm{i}=\mathrm{j} \text { and } \mathrm{i} \in \mathrm{T} \\ 0, & \text { if } \mathrm{i} \neq \mathrm{j}\end{cases}
$$

$$
A_{t i j}= \begin{cases}-\frac{1}{Y_{m I}^{\prime}}, & \text { if } \mathrm{i} \neq \mathrm{j} \text { and } i=j-1 \\ 0, & \text { elsewhere. }\end{cases}
$$

Moreover, $n \times 1$ vectors $\mathbf{J}$ and $\mathbf{E}$ contain all loop currents and transformed modal voltage sources corresponding to external field interference as indicated by (13) and (14), respectively

$$
\begin{aligned}
\mathbf{J} & =\left[\begin{array}{c}
J_{1} \\
J_{2} \\
\vdots \\
J_{n}
\end{array}\right] \\
\mathbf{E} & =\left[\begin{array}{c}
\int_{0}^{h} E_{z_{m I}}^{e}(0, z) d z+E_{x m I}^{e}(x, h) d x \\
E_{x m I}^{e}(x, h) d x \\
\vdots \\
-\int_{0}^{h} E_{z m I}^{e}(L, z) d z+E_{x m I}^{e}(x, h) d x
\end{array}\right] .
\end{aligned}
$$

After having computed the unknown vector $\mathbf{J}$ using (9), the modal noise voltage at the receiving device installed at the end of the examined transmission line can be calculated as the voltage drop across its impedance, that is, the termination impedance $Z_{b}$ in Fig. 1 as shown in (15). For the voltage signal corresponding to the actual noise, this procedure has to be repeated for all $N$ modes and the modal quantities have to be transformed by implementing the proper transformation as indicated by

$$
\begin{aligned}
V_{n m I} & =J_{n} \cdot Z_{b} \\
\mathbf{V}_{\mathbf{n}} & =\mathbf{T}_{\mathbf{V}} \cdot \mathbf{V}_{\mathbf{n} \mathbf{m}} .
\end{aligned}
$$

\section{Noise Calculation at Topologies With Lateral Branches}

Topologies that contain lateral branches require a slightly different approach compared to the topologies with no branches. The mesh current method can be used for this case too. The $n$ loops that are formed are numbered starting from the point where the transmitting communication device is installed and ending at the point where the receiving device is installed as shown in Fig. 2, in which the transmitting device is considered to be installed at loop 1, whereas the receiving device is at loop $n$. The specific topology includes a lateral branch, terminated to impedance $Z_{\text {term }}$, that is perpendicular to the main direction of the transmission line and because of that, the effect of the external EM field to the loops of the branch is taken into consideration by the addition of the $E_{y_{m I}}^{e}(y, h) d y$ voltage sources. The set $T$, as was defined for the no-branches case, is needed. Furthermore, for a set $J$ containing the number of loops from which the lateral branches start, a set $B L$ containing 


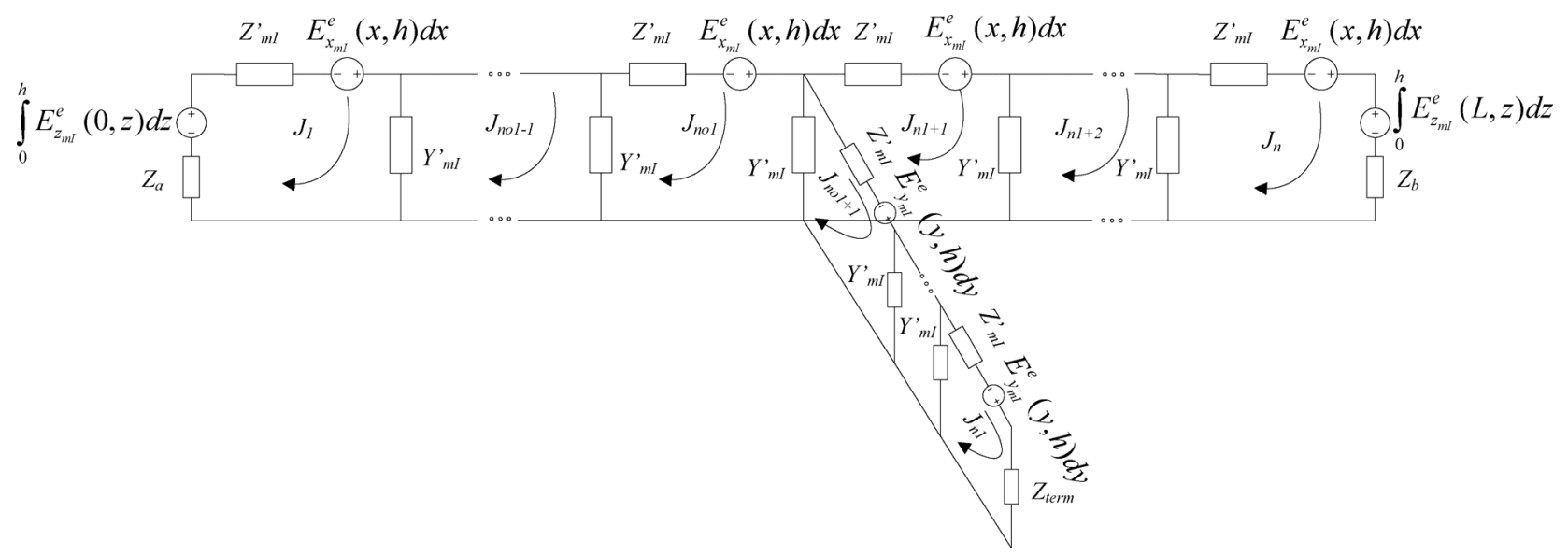

Fig. 2. Mesh current method for the $I$ th mode of a topology with one lateral branch.

the corresponding lengths of the lateral branches and a set $B N$ containing the loop numbers that correspond to lateral branches need to be defined. For example, referring to the case shown in Fig. 2, the sets that need to be introduced are $T=\left\{1, n_{1}, n\right\}$, $J=\left\{n_{o 1}\right\}, B L=\left\{n_{1}-n_{o 1}\right\}$, and $B N=\left\{\left[n_{o 1+1}, n_{1}\right]\right\}$.

Let us consider a topology that contains one branch that starts from loop $n_{o 1}$ of the transmission line. The numbering of the loops is shown in Fig. 2 and the application of the mesh current method for loop $n_{o 1}$ is shown in (17). Since it is deducted at the starting points of branches, four loop currents are involved instead of two that had to be taken into account for topologies with no branches

$$
\begin{array}{r}
-\frac{1}{Y_{m I}^{\prime}} \cdot J_{n_{o 1}-1}+\left(Z_{m I}^{\prime}+\frac{2}{Y_{m I}^{\prime}}\right) \cdot J_{n_{o 1}}-\frac{1}{Y_{m I}^{\prime}} \cdot J_{n_{o 1}+1-} \\
-\frac{1}{Y_{m I}^{\prime}} \cdot J_{n_{1}+1}=E_{x_{m I}}^{e}(x, h) d x .
\end{array}
$$

For that reason, the $n \times n$ matrix $\mathbf{A}$ is calculated as

$$
\mathbf{A}=\mathbf{A}_{\mathbf{d}}+\mathbf{A}_{\mathbf{t}}+\left(\mathbf{A}_{\mathbf{t}}\right)^{t}+\mathbf{A}_{\mathbf{k}}+\left(\mathbf{A}_{\mathbf{k}}\right)^{t}
$$

where the elements of the matrix $\mathbf{A}_{\mathbf{k}}$ are derived as shown in (19), and $B L_{i}$ is the value of the BL set that corresponds to the branch that starts from the $i$ th loop. Matrices $\mathbf{A}_{\mathbf{d}}$ and $\mathbf{A}_{\mathbf{t}}$ are used as defined in Subsection III-B

$$
A_{k i j}= \begin{cases}-\frac{1}{Y_{m I}^{\prime}}, & \text { if } \mathrm{i} \in \mathrm{J} \text { and } j=i+B L_{i}+1 \\ 0, & \text { elsewhere }\end{cases}
$$

Moreover, the vector $\mathbf{E}$ can be calculated as the sum of vectors $\mathbf{E}_{\mathbf{h}}$ and $\mathbf{E}_{\mathbf{v}}$ as in (20), where $\mathbf{E}_{\mathbf{h}}$ and $\mathbf{E}_{\mathbf{v}}$ are defined in (21) and (22), respectively, as follows:

$$
\begin{aligned}
\mathbf{E} & =\mathbf{E}_{\mathbf{h}}+\mathbf{E}_{\mathbf{v}} \\
\mathbf{E}_{\mathbf{h}} & =\left[\begin{array}{c}
\int_{0}^{h} E_{z m I}^{e}(0, z) d z+E_{x m I}^{e}(x, h) d x \\
E_{x m I}^{e}(x, h) d x \\
\vdots \\
-\int_{0}^{h} E_{z_{m I}}^{e}(L, z) d z+E_{x m I}^{e}(x, h) d x
\end{array}\right] \\
E_{v i 1} & = \begin{cases}E_{y_{m I}^{e}}^{e}(y, h) d y-E_{x m I}^{e}(x, h) d x, & \text { if } \mathrm{i} \in \mathrm{BN} \\
0, & \text { elsewhere. }\end{cases}
\end{aligned}
$$

After having formulated the $n \times n$ matrix $\mathbf{A}$ and vector $\mathbf{E}$, the unknown vector $\mathbf{J}$ can be computed by applying (23). Finally, the modal noise voltage can be computed by using (15) and (16), as in the case with no lateral branches

$$
\mathbf{J}=\mathbf{A}^{-1} \cdot \mathbf{E} .
$$

\section{Transmission-Line Capacity for PLC Systems}

The data transmission capacity of power lines is the maximum transmission rate over a channel, regardless of modulation or any other system considerations [12] and is a crucial characteristic that may determine the amount and features of the services that may be offered. Capacity is mainly affected by four key elements, namely: 1) the available frequency range; 2) the total injected power; 3) the channel transfer function; and 4) the noise present in the communication channel. Equation (24) expresses, in mathematical terms, the total available capacity $c$ of a communication channel by applying the water filling algorithm in the frequency domain as shown in [12] and [30]

$$
c=\int_{f_{\text {down }}}^{f_{\mathrm{up}}} \frac{1}{2} \log _{2}\left[1+\frac{\left(p-\frac{N_{0}(f)}{2 \mid H(f)^{\prime 2}}\right)^{+}}{\frac{N_{0}(f)}{2|H(f)|^{2}}}\right] d f .
$$

In this equation, $f_{\text {down }}$ and $f_{\text {up }}$ are the minimum and maximum frequencies of the given range, respectively; $p$ is the injected power at each distinct frequency; $H(f)$ is the channel transfer function; and $N_{0}(f)$ is the noise level at frequency $f$. Moreover, the notation $[X]^{+}$indicates the maximum value between quantity $X$ and zero. In addition, the total injected power $P$ over the total available frequency range is given by

$$
P=\int_{f_{\text {down }}}^{f_{\text {up }}}\left(p-\frac{N_{0}(f)}{2|H(f)|^{2}}\right)^{+} d f .
$$

The noise power spectral density (PSD), expressed as $N_{0}(f)$ in the aforementioned equations, may be determined by applying the proposed model using as an input the properties of the external EM field. Concerning the transfer function computations, the hybrid Smith chart (HSC) method was selected for the modal transfer function derivations [31] (i.e., $H_{m}(f)$ in this 
work), as shown in (26), due to the fact that it is easily implemented in source programming code

$$
H_{m}(f)=\prod_{k=1}^{M+1} \frac{\left(1+p_{L m}^{k}\right) e^{-\gamma_{m} L_{k}}}{1+p_{L m}^{k} e^{-2 \gamma_{m} L_{k}}} .
$$

In this equation, $p_{L m}^{k}$ corresponds to the mode coefficients of the reflections that a signal is subjected to along its path from the transmitting to the receiving device for the studied mode, $\gamma_{m}$ is the propagation constant of the studied mode, and $L_{k}$ is one of the total $M+1$ lengths of line segments between two successive reflections across the path to the receiver as explained in [7] and [31]. The transfer functions of all existing modes form a diagonal matrix $H_{m}$. Moreover, the transfer function matrix $H$ can be calculated by applying the proper modal transformation

$$
\mathbf{H}=\mathbf{T}_{\mathbf{V}} \mathbf{H}_{\mathbf{m}} \mathbf{T}_{\mathbf{V}}^{-1} .
$$

\section{Simulations}

\section{A. Baseline Scenario}

The vast extent of the installed, overhead MV networks contains a variety of network topologies. However, for the investigation of the properties of a broadband PLC system, it is sufficient to study the network segment between two adjacent communication devices, corresponding to the typical PLC repeater span of $1 \mathrm{~km} \mathrm{[7],} \mathrm{[12]-[15].} \mathrm{For} \mathrm{the} \mathrm{purposes} \mathrm{of} \mathrm{this} \mathrm{paper,} \mathrm{a}$ test network topology had to be selected within these limits in order to test the ability of the proposed model to calculate the induced noise on a PLC system by external EM waves. For this reason, a topology was selected, without loss of generality, with a total length of $1 \mathrm{~km}$. Three lateral branches, with lengths equal to $100,50,150 \mathrm{~m}$, respectively, are located at distances of 300 , 500 , and $600 \mathrm{~m}$, respectively, from the topology's starting point, that is, the first communication device. All branches are considered to lead to power transformers, which are regarded as open circuits within the studied high-frequency ranges. The branches are considered to be perpendicular to the main topology's direction which is considered to be parallel to the $x$ axis of the Cartesian coordinate system. Therefore, the direction of branches is parallel to the $y$ axis.

The second communication device is installed at the end of the selected topology. Furthermore, both communication devices are matched to the transmission line's characteristic impedance. The geometric configuration of the overhead MV transmission line comprises three ACSR phase wires of a $70 / 12-\mathrm{mm}^{2}$ cross section, located at a height of $10 \mathrm{~m}$ above the surface of the earth, in a symmetrical horizontal arrangement. Specifically, the outer conductors have 1-m spacing from the middle one, as shown in Fig. 3.

The earth, considered for the simulations, has a resistivity equal to $100 \Omega \mathrm{m}$ and a relative permittivity equal to 10 . Moreover, if it is not stated differently, the total injected power from the communication devices is equal to $5 \mathrm{dBmW}$, and the studied external plane EM-field propagation forms a 40 degree angle with the $x$ axis of the $x z$ plane, and a 20 degree angle with the $y$

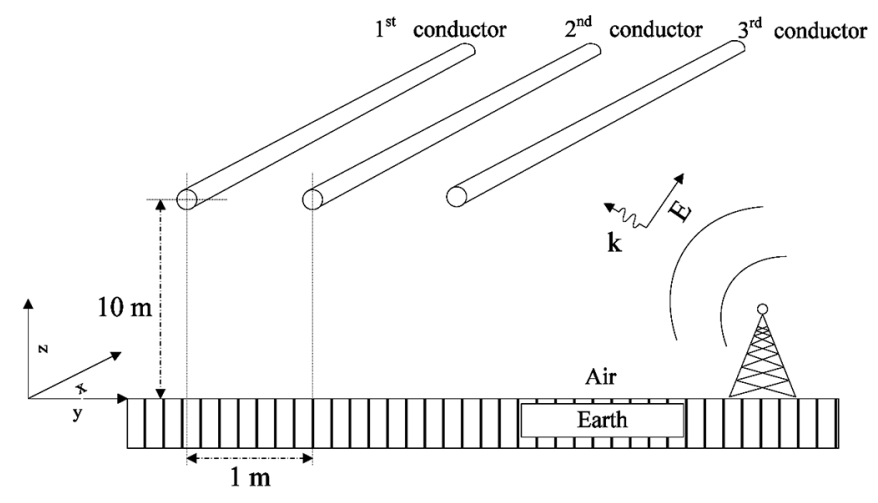

Fig. 3. Configuration of three wires above a lossy ground plane, in the presence of an external EM field with propagation vector $\mathrm{k}$.

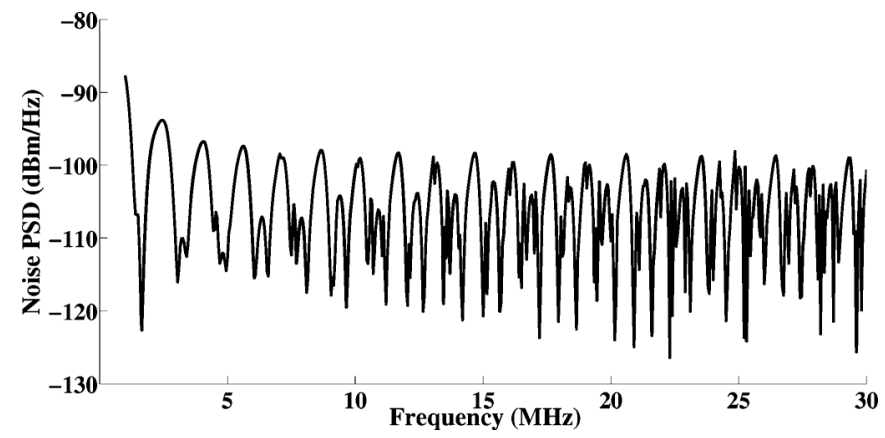

Fig. 4. Noise PSD versus frequency induced by an external field with a magnitude of $0 \mathrm{~dB} \mu \mathrm{V} / \mathrm{m}$.

axis of the $x y$ plane. The directions of the $x y z$ Cartesian coordinate system are shown in Fig. 3. Moreover, the chosen generic amplitudes of the examined EM fields are considered to correspond to the same value for all examined frequencies.

\section{B. PSD Calculations}

In Fig. 4, the power spectral density (PSD) of the noise induced by an external EM field of magnitude equal to $0 \mathrm{~dB} \mu \mathrm{V} / \mathrm{m}$ is plotted versus frequency. The noise is calculated at the point where the second communication device is considered to be located. It is obvious that the noise PSD exhibits a nonstationary behavior over the studied frequency range, in contrast with the constant level model that suggests a static level of noise throughout the entire range. The knowledge of the exact noise pattern may be exploited by advanced schemes that could be implemented for the optimum transmitted power allocation. These schemes would aim to optimize the usage of the available frequency range for the maximization of the channel capacity, allow transmission of higher power at frequencies where the noise exhibits low values, while, on the other hand, transmitting with lower power at frequencies with high noise values. In Fig. 4, it may be observed that the noise PSD varies from approximately -85 to $-125 \mathrm{dBm} / \mathrm{Hz}$.

In Fig. 5, the results are presented concerning the same configuration, with a difference in the magnitude of the external field which, in this case, is equal to $-20 \mathrm{~dB} \mu \mathrm{V} / \mathrm{m}$. The plotted curve follows the same pattern with the previous examined case, 


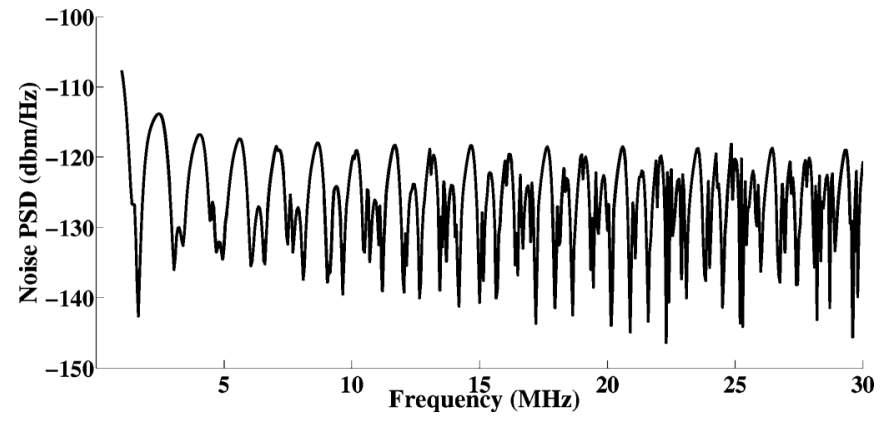

Fig. 5. Noise PSD versus frequency induced by an external field with a magnitude of $-20 \mathrm{~dB} \mu \mathrm{V} / \mathrm{m}$.

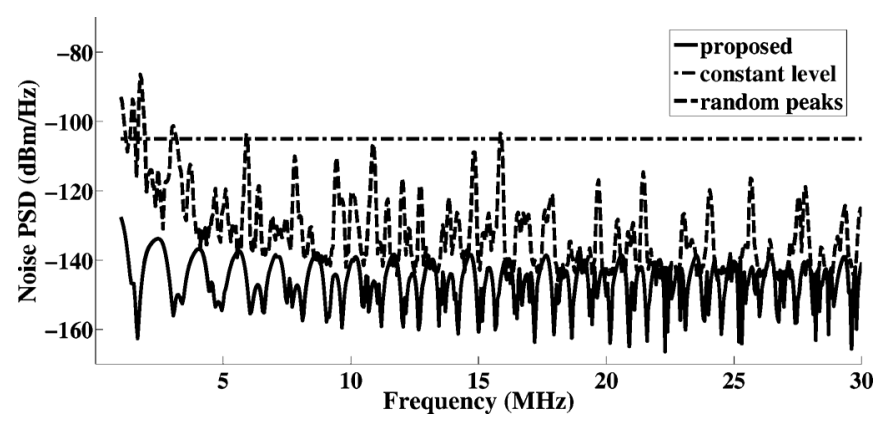

Fig. 6. Comparison between noise PSD versus frequency derived from the proposed, constant level, and random peaks' models.

as was expected, but exhibits lower values, as a result of the decreased occurring source of origin (i.e., the EM field). It may be observed that the noise PSD varies in this case from approximately -105 to $-145 \mathrm{dBm} / \mathrm{Hz}$. The most interesting conclusion with respect to these figures is that the calculated noise may be easily attributed to a specific EM-field value with the application of the proposed model. This offers a significant advantage to the proposed method compared to the existing models in the literature, because the noise and, as a result, the capacity of a PLC channel may be calculated under different cases of EM interference (EMI), leading to a comprehensive evaluation of its effect on the system operation.

The difference in the results of the proposed model compared to models that may be found in the literature is shown in Fig. 6. More specifically, the case that corresponds to an external EM field of $-40 \mathrm{~dB} \mu \mathrm{V} / \mathrm{m}$, is presented in Fig. 6 along with two existing models (i.e., the constant level model and the random peaks model). The value used in the constant level model is $-105 \mathrm{dBm} / \mathrm{Hz}$, as a proper value suggested in the literature. It may be noticed that this value is approximately equal to the mean value of the PSD derived by the proposed method, that corresponds to the examined case shown in Fig. 4 (concerning an external field with a magnitude of $0 \mathrm{~dB} \mu \mathrm{V} / \mathrm{m}$ ). The random peaks' model contains narrowband peaks corresponding to the interference from the surrounding area without providing any other information regarding the EM fields producing them. Moreover, this model was initially introduced for the calculation of noise on LV installations and, as may be observed, does not take into account the specific characteristics of the overhead MV network. On the contrary, the model proposed here may be utilized by incorporating various EM magnitudes

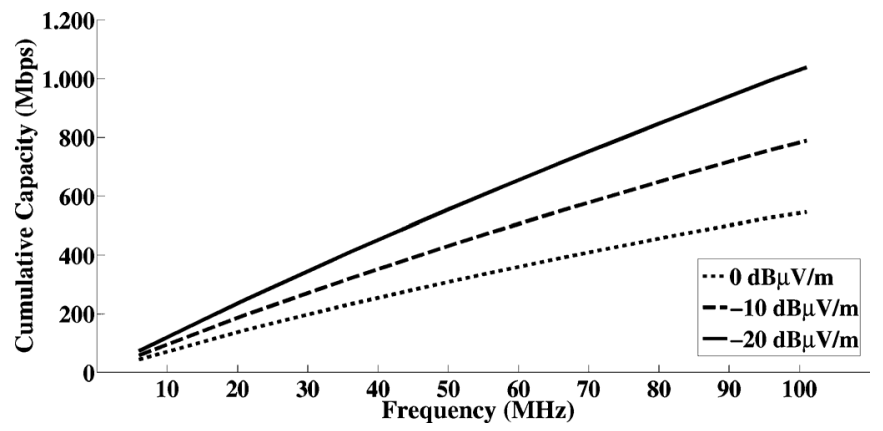

Fig. 7. Cumulative capacity versus the available frequency range for different magnitudes of the external field.

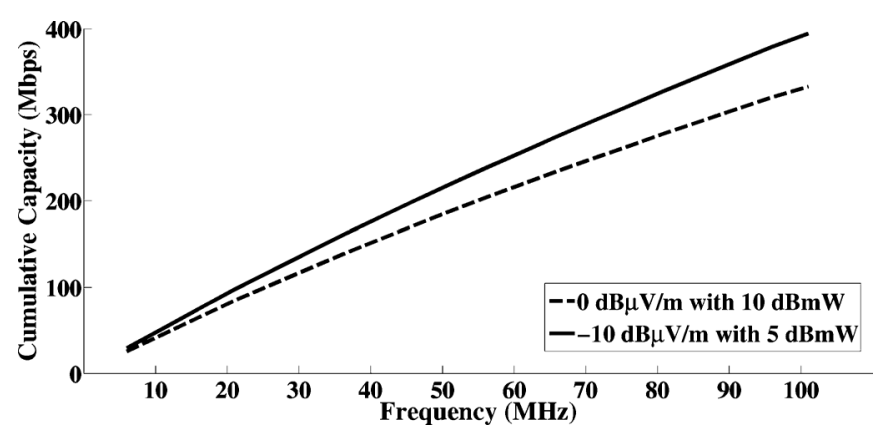

Fig. 8. Cumulative capacity versus the available frequency range for different magnitudes of the external field and transmitted power.

or directions of propagation, and may provide more accurate results for each examined case.

\section{Cumulative Capacity Calculations}

In Fig. 7, the cumulative capacity of the PLC channel is plotted versus the available frequency range for three different cases of the external EM field, with magnitudes of $0,-10$, and $-20 \mathrm{~dB} \mu \mathrm{V} / \mathrm{m}$, respectively. It may be easily derived that low-field magnitudes cause low-noise PSD and, therefore, result in high available capacity. The highest observed values of cumulative capacity for these three cases are 1038, 788.5, and 545.9 Mbps, respectively, appearing naturally at the maximum frequency range considered (i.e., $100 \mathrm{MHz}$ ). The figure content indicates the importance of the EMI that affects the induced transmission line's noise and through that the overall channel's data capacity. Moreover, the channel capacity is a crucial element that may determine the amount and type of services that a PLC system may provide.

Since the cumulative capacity is reduced due to the increase of the external EM-field magnitude, the total injected transmission power could be proportionally increased as a means to maintain the capacity level. In Fig. 8, the increase of the total injected power and its influence to the total capacity is examined. From the comparison between Figs. 7 and 8, it may be derived that a $100 \%$ increase in the total transmitted power leads to a $22 \%$ increase in the total available capacity for a $0 \mathrm{~dB} \mu \mathrm{V} / \mathrm{m}$ external field. At the same time, this increase produces a lower cumulative capacity compared to the case of a $-10 \mathrm{~dB} \mu \mathrm{V} / \mathrm{m}$ external field combined with a total transmitted power of $5 \mathrm{dBmW}$.

Moreover, in Fig. 9, comparative results are shown regarding the cumulative channel capacity for an available frequency 


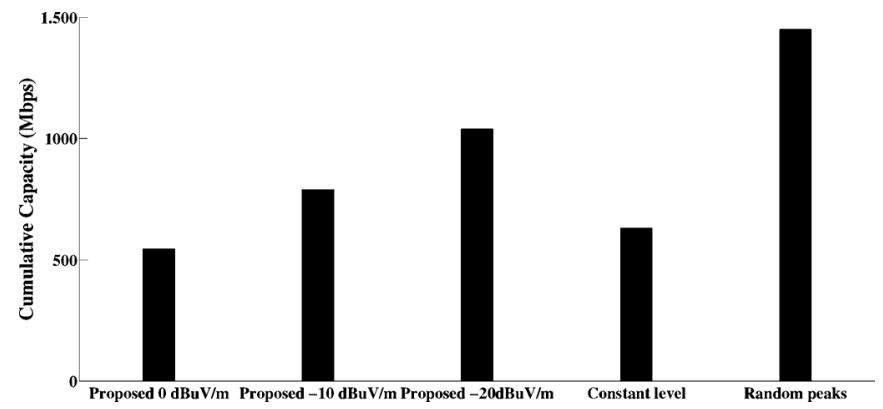

Fig. 9. Cumulative capacity calculated for different noise models for an available bandwidth up to $100 \mathrm{MHz}$.

range from 1 to $100 \mathrm{MHz}$, for different EM-field levels calculated using the proposed model, as well as the other two existing models. The proposed model is used for three different noise-level calculations, namely, $0,-10$, and $-20 \mathrm{~dB} \mu \mathrm{V} / \mathrm{m}$. The constant level model utilizing a unique value of noise PSD throughout the entire examined frequency range (i.e., $-105 \mathrm{dBm} / \mathrm{Hz}$ ) results in cumulative data capacity close to the one calculated with the proposed model corresponding to the external EM field of $0 \mathrm{~dB} \mu \mathrm{V} / \mathrm{m}$. This reveals the inability of the constant level model to deal with a variety of noise cases. It may also be observed that the random peaks' model produces greater capacity among the three methods. This is expected since this model takes into account only narrowband interference at a small number of bands into the selected range. Consequently, the method proposed here, combined with the knowledge of the EM activity in the surrounding area of an installed PLC system, may provide important information for the exact cumulative capacity estimation.

\section{Calculations Regarding Standards and Proposed Emission Levels}

The emissions of PLC and other systems utilizing the same frequency range are of high importance and should be compliant with specific standards ensuring the EMC between various installations. Until today, there have been several proposals from different national and international organizations concerning the allowed emissions of PLC systems, such as the British Broadcasting Corporation (BBC), NATO, Federal Communications Commission (FCC), Germany, and Norway, but their effects have not been thoroughly examined. Furthermore, the suggested emission levels differ from each other significantly, resulting in substantially different EM activity situations. These standards and recommendations focus on the highest value of the electric field that may originate from a communication system using the selected frequency range at a specific distance from the arrangement under study. In [32], the levels proposed by the different standards are shown in a common diagram referring to the allowed emissions, measured at a distance of $3 \mathrm{~m}$ from the arrangement under study. Since this distance is considered to be quite small for practical cases, the results illustrated in Fig. 10 are calculated using the proposed model, as a percentage ratio among the capacity calculated for each proposal and the one computed for an external EM field of $0 \mathrm{~dB} \mu \mathrm{V} / \mathrm{m}$. Moreover, the calculations were conducted for an available frequency range

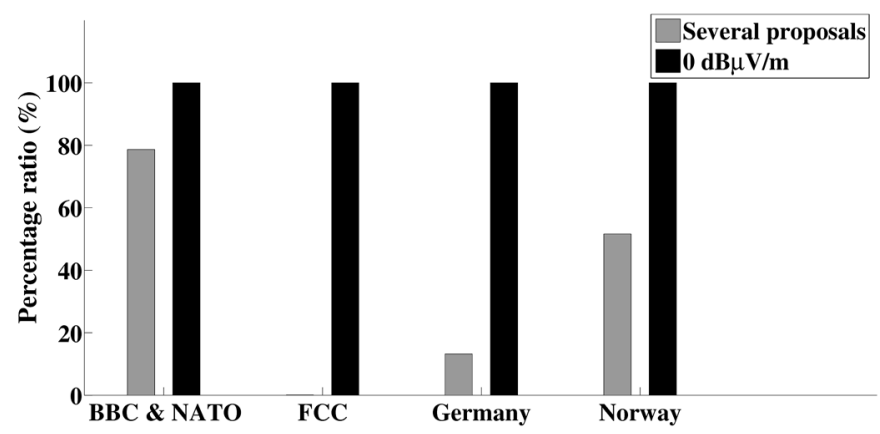

Fig. 10. Cumulative capacities for noise levels corresponding to different standards, compared to the capacity derived in the presence of a $0 \mathrm{~dB} \mu \mathrm{V} / \mathrm{m}$ external field.

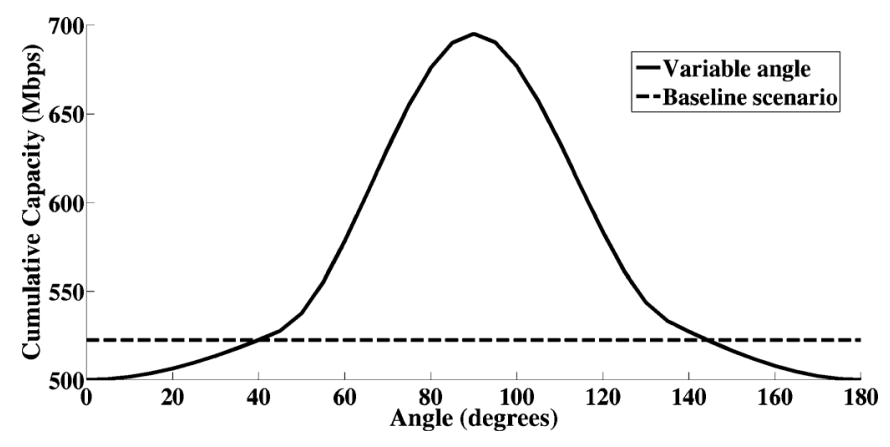

Fig. 11. Cumulative capacities for different angles between the power line and the direction of an external field of $0 \mathrm{~dB} \mu \mathrm{V} / \mathrm{m}$ ( $x$ axis of the $x z$ plane)

up to $30 \mathrm{MHz}$, because some of the standards did not provide values up to $100 \mathrm{MHz}$. For each case, the induced noise calculations were conducted, assuming that the EM activity at the vicinity of the transmission line is determined by each standard. Conclusively, the emissions proposed by BBC and NATO lead to the highest cumulative capacity. On the contrary, the FCC proposal could create an EM environment of high noise. This could cause problems for other existing systems by deteriorating their performance significantly. This comes as a result of the core idea behind the specific standard, that focuses on permitting the systems to transmit large amounts of power, without taking into account the EMC problems that may be caused to adjacent installations.

\section{E. Influence of the EM-Field Direction}

The investigation of different angles between the external EM field and the transmission line, as well as the respective effect on the available capacity, also appears to be interesting. For that reason, in Fig. 11, the computed cumulative capacity of a power line is plotted, for an external EM field with a magnitude equal to $0 \mathrm{~dB} \mu \mathrm{V} / \mathrm{m}$, and with a direction that results in different angles with the line regarding the $x$ axis of the $x z$ plane. In this figure, the capacity of the initial test case is included as the baseline scenario, corresponding to a $40^{\circ}$ angle. It may be concluded that the examined angle has a substantial effect on the overall capacity. More specifically, a divergence of approximately $400 \mathrm{Mb} / \mathrm{s}$ is observed between the perpendicular and parallel field incidence, with the former resulting naturally in the highest capacity value. Expressed in percentage ratio terms, this deviation is equal to $78 \%$, a result indicating that the knowledge of the external field 


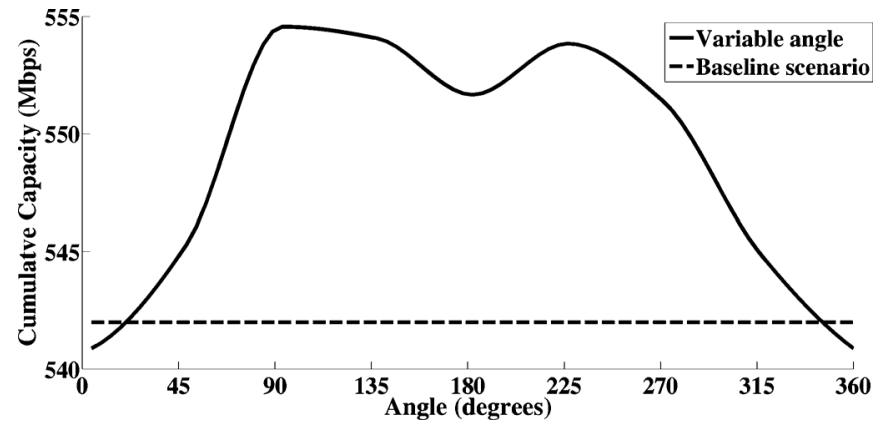

Fig. 12. Cumulative capacities for different angles between the power line and the direction of an external field of $0 \mathrm{~dB} \mu \mathrm{V} / \mathrm{m}$ ( $y$ axis of the $x y$ plane).

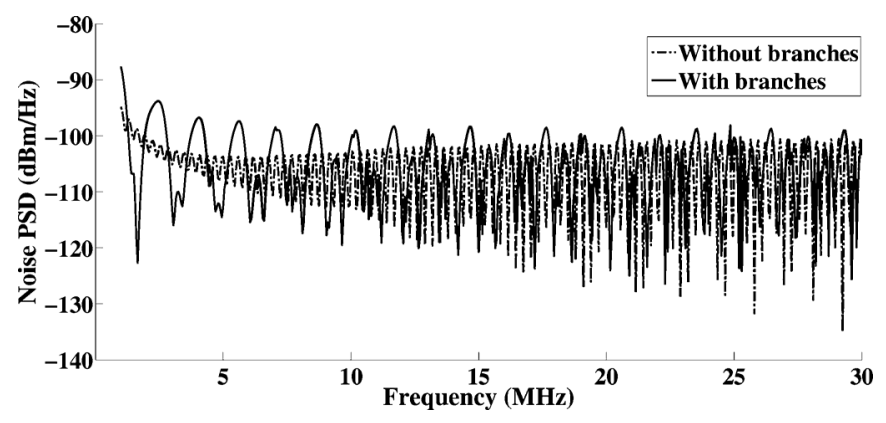

Fig. 13. Noise PSD versus frequency induced on topologies with and without branches by an external field with a magnitude of $0 \mathrm{~dB} \mu \mathrm{V} / \mathrm{m}$.

direction of propagation is of high importance. On the contrary, a possible angle between the external EM-field direction and the $y$ axis of the $x y$ plane seems to be insignificant in terms of the power-line data capacity. This may be observed in Fig. 12, for an external EM field of magnitude equal to $0 \mathrm{~dB} \mu \mathrm{V} / \mathrm{m}$. Again, the initial test case with a respective angle of $20^{\circ}$ is also included in this figure, corresponding to the baseline scenario. The highest deviation occurring in this case reaches approximately $15 \mathrm{Mbps}$, confirming the conclusion that this specific angle has no practical effect on the overall available channel's data capacity.

\section{F. Influence of Lateral Branches}

In addition, a comparison has been conducted, concerning the noise PSD induced on two different power-line topologies; the one studied throughout the aforementioned cases, which contains branches, and a respective one with no branches across the $1 \mathrm{~km}$ that separates the communication devices. The respective results are presented in Fig. 13. As may be easily derived, the induced noise PSD versus frequency depends on the topology of the transmission line under study and, thus, the topology has to be taken into account. More specifically, no significant deviation is observed between the two different topologies in terms of mean value over the entire selected frequency range, but there is a difference in the pattern that each of the two illustrated curves follows.

\section{G. Influence of Earth's EM Properties}

Finally, an issue that is interesting to deal with is the effect of the earth's EM properties, that is, earth resistivity $(\rho)$ and relative permittivity $\left(\varepsilon_{r}\right)$ to the overall induced noise. As was described in the section of theoretical analysis, the parameters of

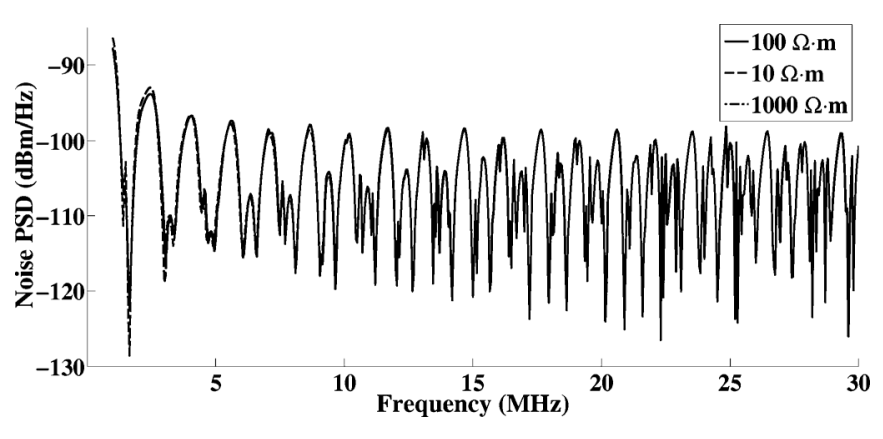

Fig. 14. Noise PSD versus frequency induced on a topology with branches by an external field with a magnitude of $0 \mathrm{~dB} \mu \mathrm{V} / \mathrm{m}$ for various values of earth resistivity.

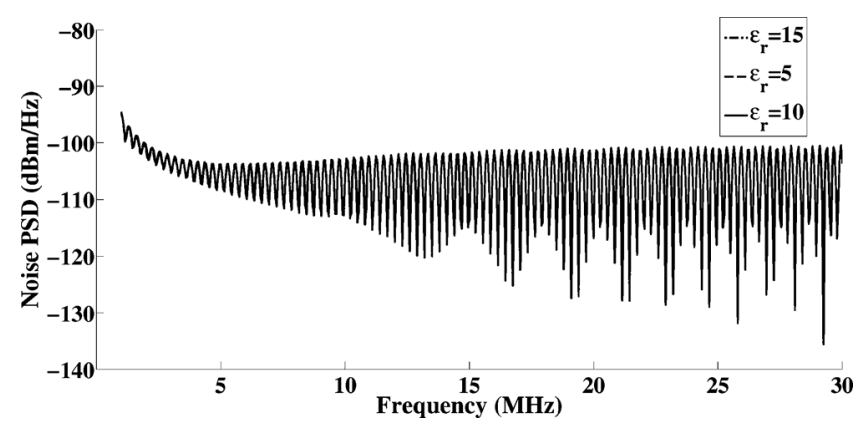

Fig. 15. Noise PSD versus frequency induced on a topology without branches by an external field with a magnitude of $0 \mathrm{~dB} \mu \mathrm{V} / \mathrm{m}$ for various values of earth permittivity.

the earth are taken into account for the per-unit length electrical characteristics computation. In Fig. 14 as well as in Fig. 15, the noise induced on a studied topology from an external EM field of $0 \mathrm{~dB} \mu \mathrm{V} / \mathrm{m}$ is calculated for various values of the earth's resistivities and permittivities, respectively. The former figure indicates that the influence of the earth's resistivity may be considered negligible, whereas the latter one indicates the same concerning the influence of the earth's permittivity. Conclusively, the noise PSD is affected mainly by the characteristics of the external EM field and the transmission-line geometrical configuration, and not by the earth's parameters.

\section{CONCLUSION}

In this paper, a novel method is presented for modeling noise induced on a PLC system installed on overhead MV power lines. Compared to other models existing in the literature, it offers the capability of taking into account several parameters of the studied configuration that may influence the results. Focusing on the nature of MV overhead power lines, the external EM fields are identified as the dominant source of noise, causing the absence of cable shielding. Different magnitudes of interfering fields are examined, and the induced noise is calculated. Moreover, their effect on the overall PLC channel cumulative capacity is computed. Noise PSD and capacity are computed using the proposed model and it is concluded that they both are considerably influenced by the magnitude and EM field's direction of propagation. Important results are also derived from the noise levels extracted from different emission levels proposed in the existing standards by several organizations which lead to 
significantly different capacities. Furthermore, the effect of the exact power-line topology and its possible branches are examined, and it is concluded that they have to be taken into account for noise calculations. Finally, the earth's EM properties have no influence on the results, leading to the conclusion that the geometrical configuration of the arrangement and the characteristics of the external field are the key parameters for the noise estimation procedure.

\section{REFERENCES}

[1] S. Galli, A. Scaglione, and Z. Wang, "For the grid and through the grid: The role of power line communications in the smart grid," Proc. IEEE, vol. 99, no. 6, pp. 998-1027, Jun. 2011.

[2] W. Chen, Home Networking Basis: Transmission Environments and Wired/Wireless Protocols. Upper Saddle River, NJ: Prentice-Hall, 2003.

[3] H. Ferreira, L. Lampe, J. Newbury, and T. Swart, Power Line Communications: Theory and Applications for Narrowband and Broadband Communications Over Power Lines. Hoboken, NJ: Wiley, 2010.

[4] J. Anatory and N. Theethayi, Broadband Power Line Communications Systems: Theory and Applications. Southampton, U.K.: WIT Press/ Computational Mechanics, 2010.

[5] H. Hrasnica, A. Haidine, and R. Lehnert, Broadband Powerline Communications Networks: Network Design. Hoboken, NJ: Wiley, 2004.

[6] P. Amirshahi, S. Navidpour, and M. Kavehrad, "Performance analysis of uncoded and coded ofdm broadband transmission over low voltage power-line channels with impulsive noise," IEEE Trans. Power Del., vol. 21, no. 4, pp. 1927-1934, Oct. 2006.

[7] A. Lazaropoulos and P. Cottis, "Transmission characteristics of overhead medium-voltage power-line communication channels," IEEE Trans. Power Del., vol. 24, no. 3, pp. 1164-1173, Jul. 2009.

[8] J. Anatory and N. Theethayi, "On the efficacy of using ground return in the broadband power-line communications; A transmission-line analysis," IEEE Trans. Power Del,, vol. 23, no. 1, pp. 132-139, Jan. 2008.

[9] J. Anatory, N. Theethayi, and R. Thottappillil, "Power-line communication channel model for interconnected networks-Part II: Multiconductor system," IEEE Trans. Power Del., vol. 24, no. 1, pp. 124-128, Jan. 2009.

[10] J. Anatory, M. M. Kissaka, and N. H. Mvungi, "Channel model for broadband power-line communication," IEEE Trans. Power Del., vol. 22, no. 1, pp. 135-141, Jan. 2007.

[11] J. Anatory, N. Theethayi, and R. Thottappillil, "Channel characterization for indoor power-line networks," IEEE Trans. Power Del., vol. 24, no. 4, pp. 1883-1888, Oct. 2009.

[12] P. Amirshahi and M. Kavehrad, "High-frequency characteristics of overhead multiconductor power lines for broadband communications," IEEE J. Sel. Areas Commun., vol. 24, no. 7, pp. 1292-1303, Jul. 2006.

[13] P. Amirshahi and M. Kavehrad, "Medium voltage overhead power-line broadband communications; transmission capacity and electromagnetic interference," in Proc. Int. Symp. Power Line Commun. Appl., Apr. 2005, pp. 2-6.

[14] A. Lazaropoulos and P. Cottis, "Capacity of overhead medium voltage power line communication channels," IEEE Trans. Power Del., vol. 25, no. 2, pp. 723-733, Apr. 2010.

[15] J. Anatory, N. Theethayi, R. Thottappillil, M. Kissaka, and N. Mvungi, "Broadband power-line communications: The channel capacity analysis," IEEE Trans. Power Del., vol. 23, no. 1, pp. 164-170, Jan. 2008.

[16] Z. Tao, Y. Xiaoxian, Z. Baohui, C. Jian, Y. Zhi, and T. Zhihong, "Research of noise characteristics for 10-kv medium-voltage power lines," IEEE Trans. Power Del., vol. 22, no. 1, pp. 142-150, Jan. 2007.

[17] J. Lee, S. Choi, H. Oh, W. Lee, K. Kim, and D. Lee, "Measurements of the communications environment in medium voltage power distribution lines for wide-band power line communications," in Proc. 8th Int. Symp. Power-Line Commun. Appl., Zaragoza, Spain, 2004, pp. 69-74.

[18] M. Zimmermann and K. Dostert, "Analysis and modeling of impulsive noise in broad-band powerline communications," IEEE Trans. Electromagn. Compat., vol. 44, no. 1, pp. 249-258, Feb. 2002.

[19] N. Andreadou and F.-N. Pavlidou, "PLC channel: Impulsive noise modelling and its performance evaluation under different array coding schemes," IEEE Trans. Power Del., vol. 24, no. 2, pp. 585-595, Apr. 2009.

[20] N. Andreadou and F.-N. Pavlidou, "Modeling the noise on the OFDM power-line communications system," IEEE Trans. Power Del., vol. 25, no. 1, pp. 150-157, Jan. 2010.

[21] M. Loftness, AC Power Interference Handbook, 3rd ed. Newington, CT, USA: ARRL, 2003

[22] E. Kuffel, W. Zaengl, and J. Kuffel, High Voltage Engineering: Fundamentals. Oxford, U.K.: Newnes, 2000.

[23] A. Agrawal, H. Price, and S. Gurbaxani, "Transient response of multiconductor transmission lines excited by a nonuniform electromagnetic field," IEEE Trans. Electromagn. Compat., vol. EMC-22, no. 2, pp. 119-129, May 1980.

[24] F. Rachidi, "Formulation of the field-to-transmission line coupling equations in terms of magnetic excitation field," IEEE Trans. Electromagn. Compat., vol. 35, no. 3, pp. 404-407, Aug. 1993.

[25] F. Rachidi and S. Tkachenko, Electromagnetic Field Interaction With Transmission Lines: From Classical Theory to HF Radiation Effects. Southampton, U.K.: WIT Press/Computational Mechanics, 2008, vol. 5.

[26] M. D'Amore and M. Sarto, "A new formulation of lossy ground return parameters for transient analysis of multiconductor dissipative lines," IEEE Trans. Power Del., vol. 12, no. 1, pp. 303-314, Jan. 1997.

[27] C. Paul, Analysis of Multiconductor Transmission Lines. Hoboken, NJ: Wiley/IEEE, 2008.

[28] L. Wedepohl, "Application of matrix methods to the solution of travelling-wave phenomena in polyphase systems," Proc. Inst. Elect. Eng., vol. 110, no. 12, pp. 2200-2212, Dec. 1963.

[29] W. Hayt, J. Kemmerly, and S. Durbin, Engineering Circuit Analysis. New York: McGraw-Hill, 1986.

[30] T. Cover and J. Thomas, Elements of Information Theory 2nd Edition (Wiley Series in Telecommunications and Signal Processing). Hoboken, NJ: Wiley, 2006.

[31] H. Meng, S. Chen, Y. Guan, C. Law, P. So, E. Gunawan, and T. Lie, "Modeling of transfer characteristics for the broadband power line communication channel," IEEE Trans. Power Del., vol. 19, no. 3, pp. 1057-1064, Jul. 2004.

[32] "HF Interference, procedures and tools, final rep. of NATO RTO information systems technology RTO-TR-ISTR-050," NATO Tech. Rep., Jun. 2007. [Online]. Available: http://ftp.rta.nato.int/public/PubFullText/RTO/TR/RTO-TR-IST-050/\$\$TR-IST-050-ALL.pdf

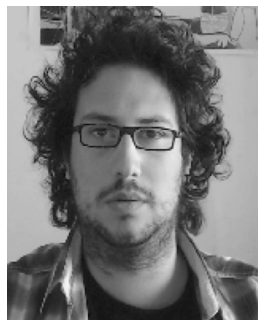

communications.
Apostolos N. Milioudis (S'09) was born in Xanthi, Greece, on February 27, 1985. He received the Dipl.Eng. and Ph.D. degrees in electrical and computer engineering from the Aristotle University of Thessaloniki, Thessaloniki, Greece, in 2007 and 2012, respectively.

His special interests are power system analysis with a special emphasis on the simulation of transmission and distribution systems, high-impedance fault detection techniques, artificial-intelligence applications in power systems, and power-line

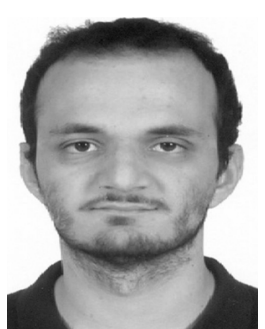

Konstantinos N. Syranidis was born in Thessaloniki, Greece, on August 10, 1987. He received the Dipl.-Eng. degree in electrical and computer engineering from the Aristotle University of Thessaloniki, Thessaloniki, Greece, in 2011.

His special interests are power systems analysis and power-line communications. 


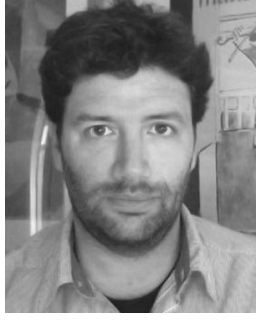

Georgios T. Andreou (S'98-A'02-M'08) was born in Thessaloniki, Greece, on August 16, 1976. He received the Dipl.-Eng. and the Ph.D. degrees in electrical and computer engineering from the Aristotle University of Thessaloniki, Thessaloniki, Greece, in 2000 and 2006, respectively.

Currently, he is a Lecturer at the Aristotle University of Thessaloniki. His special interests are power system analysis with a special emphasis on the simulation of transmission and distribution systems, electromagnetic- and thermal-field analysis, and power-

line communications

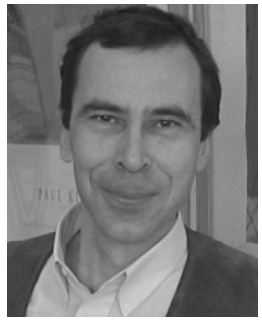

Dimitrios P. Labridis (S'88-M'90-SM'00) was born in Thessaloniki, Greece, on July 26, 1958. He received the Dipl.-Eng. and $\mathrm{Ph} . \mathrm{D}$. degrees in electrical and computer engineering from the Aristotle University of Thessaloniki, Thessaloniki, Greece, in 1981 and 1989, respectively.

Since 1990, he has been with the Electrical Engineering Department, Aristotle University of Thessaloniki, where he is currently a Professor. His research interest is power system analysis with a special emphasis on the simulation of transmission and distri- 